\title{
Mechanisms of inhibitory discriminative control
}

\author{
CHARLOTTE BONARDI \\ University of Cambridge, Cambridge, England
}

\begin{abstract}
Rats were trained to discriminate between trials signaled by a tone, during which leverpressing was reinforced with food, and trials signaled by the tone in compound with a light stimulus, during which no reinforcers were delivered. A subsequent transfer test suggested that the light had acquired the ability to suppress operant responding; there was no evidence that this suppression could be attributed to Pavlovian inhibitory conditioning. It is argued that these data cannot be accommodated by current accounts of discriminative control.
\end{abstract}

A rat rewarded for pressing a lever during presentations of a tone will come to respond more in the presence of the tone than in its absence. If the tone is sometimes presented in compound with, say, a light, and on these occasions no reinforcers are delivered, the rat will learn to respond less during the tone when the light is also present. What mechanism could account for this suppressive effect of the light? Contemporary accounts of discriminative control of operant responding are provided by so-called two-factor theories (e.g., Rescorla \& Solomon, 1967; Trapold \& Overmier, 1972); these have inherited the task of explaining the phenomenon of inhibitory discriminative control (e.g., Hearst, Besley, \& Farthing, 1970; Spence, 1936). Two-factor theories are essentially stimulus-response (S-R) theories which in addition allow reinforcement to modulate instrumental responding by means of a Pavlovian association between the discriminative stimulus and the reinforcer. The twofactor account implies that a conditioned inhibitor, which by definition opposes the effects of Pavlovian excitation (Rescorla, 1969), might therefore be able to suppress the responding elicited by a discriminative stimulus. Since the tone/tone-light discrimination is effectively equivalent to a Pavlovian conditioned inhibition procedure, this account can easily be invoked to explain response suppression in this instance.

Gutman and Maier (1978) reported data suggesting that this account of response inhibition might be inadequate. They compared four groups of animals, each of which had been trained on a different multiple schedule. The schedule components were signaled by a dim houselight $\mathbf{S}+$ either alone or in compound with a white noise $\mathbf{S}-$, which was to be their target inhibitory stimulus. Animals in Group VI-Ext were rewarded for leverpressing during S+ presentations according to a variable-interval (VI)

This research was financed by grants from the Science and Engineering Research Council, the G. C. Grindley Fund, and the Cambridge Philosophical Society. I would like to thank N. J. Mackintosh for his advice throughout the course of this work, and G. Hall for helpful comments on the manuscript. Address correspondence to Charlotte Bonardi, Department of Psychology, York University, Heslington, York YOI SDD, England. 30-sec schedule, but received no reinforcement during the $\mathrm{S}+/ \mathrm{S}-$ compound. Subjects in Group VT-Ext received response-independent reinforcement delivered according to a variable-time (VT) 30-sec schedule during $S+$ presentations; they also received no reinforcers during the $S+/ S-$ compound. In both of these groups, one would therefore expect the $\mathrm{S}$ - to become a Pavlovian conditioned inhibitor, but in Group VI-Ext the $S$ - in addition signaled the absence of the response-reinforcer contingency. Animals in the control group, Group VI-VI, were rewarded for responding during both the $S+$ and the $S+/ S-$ compound. The effect of the $\mathbf{S}-$ on instrumental responding in each group was then investigated by presenting it in compound with a separately trained discriminative stimulus in an extinction test. Gutman and Maier reported that the S- suppressed instrumental responding more in Groups VI-Ext and VT-Ext than in the control Group VI-VI, and that this suppression was significantly greater in Group VI-Ext than in Group VT-Ext, even though the Pavlovian inhibitory properties of the $S$ - were supposedly the same in the two experimental groups. On the basis of these results they argued that suppression of operant responding can be produced not only by the Pavlovian properties of a stimulus, but also by properties it acquires from signaling the absence of the response-reinforcer contingency-a possibility that two-factor theory does not address.

However, interpretation of Gutman and Maier's (1978) results is complicated by reservations regarding certain aspects of the experimental procedure. First, their explanation of the results rests on the assumption that the $S-$ was an equally strong Pavlovian inhibitor in the two experimental groups, but their experimental design allowed no independent evidence that this was in fact the case. Second, to conclude that the $S$ - was suppressing responding as a result of discrimination training it is necessary to control for nonassociative disruption effects it might also produce-through, for example, generalization decrement and external inhibition. It is not clear that Group VI-VI provided an adequate control for these unconditioned effects; for animals in this group the S- signaled no change in the reinforcement contingency, and it is therefore likely that potentially disruptive responses elicited by the $\mathrm{S}-$ habituated. That the $\mathrm{S}-$ was significantly 
more suppressive in the experimental animals could therefore simply be due to the fact that the treatment given to the $S$ - in these groups had served to attenuate habituation (cf. Pfautz, Donegan, \& Wagner, 1978). There was also another experimental group, Group VI-VT, for which responding was reinforced during the $S+$ but which received response-independent reinforcement during the $\mathrm{S}+/ \mathrm{S}-$ compound. The comparison of the other experimental groups with Group VI-VT is equally problematic, however. In this group the S-, being paired with food, presumably acquired Pavlovian excitatory properties, and Pavlovian excitors have been reported in the literature to facilitate instrumental responding in extinction tests like that used in this experiment (e.g., Estes, 1943, 1948; Lovibond, 1981; Morse \& Skinner, 1958). This makes it possible that even though the $S-$ in this group might have produced as much nonassociative disruption of responding as it did in the other experimental groups, to the extent that this disruption could have been offset by a facilitation of responding produced by Pavlovian excitatory conditioning, the $\mathrm{S}-$ would not have provided an adequate control for unconditioned effects in Group VI-VT any more than in Group VI-VI.

The experiment reported here attempted to address these problems while confirming the main results of Gutman and Maier's (1978) study-that suppression of operant responding may be produced both by Pavlovian inhibitors and by stimuli that signal the absence of a responsereinforcer contingency. Three groups of animals received extinction (Ext) trials signaled by a tone-light compound during which reinforcers were never delivered. The light was the target-inhibitory stimulus and the groups differed in their experience with the tone. The two experimental groups were essentially identical to Gutman and Maier's. For subjects in Group VR, the tone signaled VR trials during which leverpressing was rewarded according to a variable ratio (VR) schedule. For subjects in Group VT, the tone signaled VT trials during which responseindependent reinforcement was delivered. Subjects in the control group, Group C, never experienced the tone alone. The first major departure from Gutman and Maier's procedure was the use of a Pavlovian retardation test, with Group $C$ as a control, to compare the level of Pavlovian inhibitory conditioning in the two experimental groups. The second was the use of a considerably more sensitive test of operant response suppression. Instead of using a between-subjects comparison, a within-subjects procedure was adopted in which the effect of the light on instrumental responding elicited by a separately trained discriminative stimulus, a clicker, was compared with the effect produced by a quite novel control stimulus. If Gutman and Maier's results were to be replicated, one would predict that the responding elicited by the clicker should be suppressed more by the light than by the novel stimulus in both experimental groups, but that the size of this effect should be greater in Group VR than in Group VT. The final difference was that a VR schedule was used in place of the VI schedule employed by Gutman and Maier. In VR schedules, increases in response rate always produce increases in reinforcement rate; in VI schedules, once the response rate has risen beyond a certain point, increases in response rate cease to produce any change in the rate of reinforcement. For this reason it has been argued that a response-reinforcer contingency may be experienced more readily with a VR schedule than with a VI schedule (Dickinson, 1985). Hence, using a VR schedule might make it easier to obtain effects that depend on the animal's detecting the absence of the response-reinforcer contingency.

The training procedure thus involved three groups of animals. In order to equate their experience, all groups were given VR, VT, and extinction trials. Group VR received VR trials signaled by the tone and extinction trials signaled by the tone-light compound, and, in addition, VT trials signaled by the noise. Group VT received VT trials signaled by the tone and extinction trials signaled by the tone-light compound, and, in addition, VR trials signaled by the noise. Finally, Group $C$ received extinction trials signaled by the tone-light compound, and, in addition, VR trials signaled by a clicker and VT trials signaled by the noise (see Table 1).

\section{METHOD}

\section{Subjects}

The subjects were 40 experimentally naive male hooded Lister rats with a mean free-feeding weight of $176 \mathrm{~g}$ (range, 165-195 g). They were housed in groups of 4 , and were maintained at $85 \%$ of their free-feeding weights by being given an hour's access to food at the end of each day's sessions.

\section{Apparatus}

The apparatus consisted of four Campden Instruments leverboxes, modified by the permanent removal of the right-hand lever and by the provision of a $60-\mathrm{W}, 240-\mathrm{V}$ strip light mounted above the white Perspex ceiling. The front panel contained a central opening to the magazine, covered by a Perspex flap door. A retractable rat lever was mounted to the left of the magazine opening. Mixed-composition 45-mg food pellets could be delivered into the magazine of each chamber. Illumination was provided by a 2.8 -W houselight wired in series with a $100-\Omega$ resistor, mounted centrally in the rear wall. The houselight was on all the time the rats were in the chambers except during the visual light-off stimulus; another visual stimulus, light-on, was provided by turning on the strip light. Above the houselight was a loudspeaker through which a $3000-\mathrm{Hz}$ tone and a white noise pulsed at approximately $2 \mathrm{~Hz}$ could be delivered. A third auditory stimulus, a clicker, was provided by operating a heavy-duty relay mounted near the front panel five times per second. All auditory stimuli were approximately $80 \mathrm{~dB}$. Masking noise was provided by the operation of the ventilating fans. The appara-

Table 1 Discrimination Training

\begin{tabular}{clll}
\hline & \multicolumn{3}{c}{ Trial Type } \\
\cline { 2 - 4 } Group & VR & VT & Ext \\
\hline VR & Tone & Noise & Tone-Light \\
VT & Noise & Tone & Tone-Light \\
C & Clicker & Noise & Tone-Light \\
\hline
\end{tabular}

Note - VR $=$ variable ratio, $\mathrm{VT}=$ variable time, $\mathrm{Ext}=$ extinction, $\mathrm{C}=$ control. 
tus was controlled by Acom Atom microcomputers programmed in ONLIBASIC.

\section{Procedure}

Preliminary training. During the first 30-min session the animals were magazine trained. The levers were removed from the chambers and reinforcers were delivered at variable intervals, with a mean of $60 \mathrm{sec}$. In the next session the levers were replaced and leverpressing was consistently reinforced until each subject had earned a minimum of 75 pellets. During the third session the subjects were preexposed to the stimuli. Leverpressing was reinforced according to a VI 30-sec schedule and the subjects were exposed to 20-sec stimulus presentations that were without programmed consequence. The tone and the clicker were each presented once, the light-on and light-off stimuli and the stimulus compounds clicker/light-on and clicker/light-off were each presented twice, and the tone in compound with the visual stimulus (light-on or light-off) with which that subject was to be trained was presented once.

From this point on, all trials were discrete, signaled, and of $20 \mathrm{sec}$ duration unless stated otherwise. All trials were separated by an intertrial interval during which no stimuli or reinforcers were presented. Each trial was always preceded by a pretrial period of the same duration as the trial (except in the initial sessions, when the trials were longer than the intertrial interval). Responses were recorded separately during each trial and each pretrial period. In order to correct for individual differences in baseline responding, the number of responses made during each trial had the number of responses made in the immediately preceding pretrial period subtracted from it. This produced a corrected score for each trial. The corrected scores for VR, VT, and Ext trials were then pooled separately to produce a measure of responding during each of these types of trial in a session.

Discrimination training. At this point the subjects were divided into three groups. Groups VR and VT were each composed of 16 subjects and Group $\mathrm{C}$ of 8 . Trials during this stage were signaled according to the plan shown in Table 1 .

The VR schedule obeyed the following constraints. First, if a subject made a number of consecutive unrewarded responses that was equal to three times the current schedule value (e.g., 18 responses for a VR-6 schedule), the next response earned a pellet. Second, no more than six pellets could be earned on any trial. Finally, in order to accommodate the yoking procedure, VR trials were programmed in 1-sec intervals. If a reward was delivered, no more reinforcers could be earned for the rest of the interval.

In Session 1 of this stage responding was reinforced according to a VR-2 schedule with an 11-sec intertrial interval, and in Session 2 it was reinforced according to a VR-3 schedule with a 21-sec intertrial interval. In subsequent sessions of discrimination training, all subjects received an equal number of VR and VT trials. Reinforcer delivery during VT trials was determined by yoking each VT trial to one of the preceding VR trials. Each subject from Group VR was a master animal, and each subject in Groups VT and $C$ was yoked to one of these master animals. Thus every subject in Group VR was yoked to a subject in Group VT, and half of the subjects in Group VR were also yoked to a subject in Group C. For each subject in Group VR, the number and temporal distribution of reinforcers earned in each VR trial were recorded. When the VT trial to which this VR trial was yoked occurred, the same pattern of reinforcer delivery was played back, both to the master animal itself and to the subjects in other groups that were yoked to it. This meant that the number of reinforcers occurring during tone trials would be the same for Group VR and Group VT, so that the level of Pavlovian inhibitory conditioning to the light should be similar in the two groups. It also ensured that all three groups would have similar experience of response-independent reinforcement.
Sessions 3-10 were programmed in pairs. The first session of each pair consisted of 14 or $16 \mathrm{VR}$ trials and the second of an equal number of VT trials. The value of the VR contingency was increased in steps from VR-3 to VR-5, and the intertrial interval from $21 \mathrm{sec}$ to $41 \mathrm{sec}$. Sessions 11-15 were composed of 14 VR and 14 VT trials. The different types of trial in each session were presented in a semirandom order. The value of the VR schedule was increased in steps from VR-3 to VR-7, but finally reduced to VR-6; it was maintained at this value for the rest of the experiment. The intertrial interval was of variable duration, with a mean of $100 \mathrm{sec}$ (range, $70-130 \mathrm{sec}$ ); unless stated otherwise, it was maintained at this value for the rest of the experiment.

The final eight sessions of discrimination training were composed of 10 VR trials and 10 VT trials, and, in addition, 10 Ext trials during which no reinforcers were ever delivered. Ext trials were signaled by the tone in compound with light-on for half the animals and light-off for the remainder. The visual stimulus not used in training was thus available for use as a novel control stimulus in the subsequent operant summation test. For ease of reference, these trials will be referred to as tone-light trials. The different types of trial were presented in a semirandom order.

Subjects that had not reached a performance criterion on the last session of discrimination training were discarded. One ratio was computed by dividing the corrected score for VT trials by that for VR trials, and a second ratio was computed by dividing the corrected score for Ext trials by that for VR trials. The lower the ratio, the less the responding during VT or Ext trials relative to VR trials, and hence the better the discrimination. Subjects for which either the VT ratio or the Ext ratio was greater than $90 \%$ during the last session of discrimination training were discarded. This left 14 subjects in Group VR, 15 in Group VT, and 8 in Group C.

Test excitor training. During the next three sessions leverpressing was reinforced during presentations of the clicker according to a VR schedule. In the first 16-trial session the value of the VR schedule was VR-2 and the intertrial interval was $40 \mathrm{sec}$. In the second 16trial session the schedule was VR-4 and the intertrial interval was $40 \mathrm{sec}$. In the final 24-trial session the schedule was VR-6, and the intertrial interval was of variable duration with a mean of $100 \mathrm{sec}$, as described above.

Operant summation test. All subjects were then given two sessions of the operant summation test. There were 24 trials in each session- 8 presentations of the clicker alone, 8 of the clicker in compound with the visual stimulus used in discrimination training, and 8 of the clicker in compound with the other, novel visual stimulus. Half of each of these types of trial were paired with a VT 14-sec schedule of response-independent reinforcement, with the additional constraint that at least one reinforcer be delivered per trial. During the other half of the trials no reinforcers were delivered. The six different types of trial were intermixed in a semirandom order.

Pavlovian retardation test. The levers were removed from the chambers and all subjects were given two sessions of a Pavlovian retardation test. Each session consisted of 12 presentations of the visual stimulus used in discrimination training. Each stimulus presentation was of $10 \mathrm{sec}$ duration, and at the offset of each presentation a single food pellet was delivered. The intertrial interval duration was increased to a mean of $120 \mathrm{sec}$ (range, $90-150 \mathrm{sec}$ ), so that the total mean (intertrial interval + trial duration) was the same as in previous phases of the experiment. The time the animals spent with the magazine flap open during trial and pretrial periods was recorded in $0.1-\mathrm{sec}$ units.

\section{RESULTS}

In all analyses reported below a significance level of $p<.05$ was adopted. Leverpressing scores were ex- 
amined using analysis of variance. Preplanned comparisons were examined with $t$ tests. Magazine entry scores showed considerable nonhomogeneity of variance and were subject to nonparametric analysis.

\section{Discrimination Training}

Response rates during the various types of trial on the last day of discrimination training for subjects meeting the performance criterion are presented in Table 2 . These data, based on corrected (i.e., trial - pretrial) scores converted into responses per minute (rpm), are presented separately for the three groups. The subjects showed a clear discrimination, responding at a higher rate during VR trials than during either VT or Ext trials.

A three-way analysis of variance with group (VR, VT, or C), type of trial (VR, VT, or Ext), and type of visual stimulus (light-on or light-off) was performed on these data. There was a significant main effect of type of trial $[F(2,62)=156.27]$. Subsequent planned contrasts using the Dunn test showed that subjects responded at a higher rate during VR trials than during either VT trials or Ext trials. There was also a significant main effect of group $[F(2,31)=8.33]$, reflecting the fact that subjects in Group VR responded at a higher rate than those in the other two groups. No other effects or interactions were significant [largest $F(2,62)=1.69$ ].

The mean pretrial response rates were $3.47,2.51$, and $2.88 \mathrm{rpm}$ for VR, VT, and Ext trials, respectively. A three-way analysis of variance with group (VR, VT, or C), type of trial (VR, VT, or Ext), and type of visual stimulus (light-on or light-off) revealed a main effect of type of trial $[F(2,62)=4.27]$. No other effects or interactions were significant [largest $F(1,31)=1.37$ ]

\section{Operant Summation Test}

Performance in the operant summation test was analyzed using suppression ratios of the form $a /(a+b)$, where $a$ is the total number of responses made during trials signaled by the clicker in compound with a visual stimulus and $b$ is the number made during trials signaled by the clicker alone. A ratio of less than 0.5 means that the visual stimulus suppressed responding elicited by the clicker. Separate ratios were calculated for test trials with each reinforcement schedule and averaged to produce, for each subject, one value for suppression produced by the visual stimulus used in training and one value for suppression produced by the novel visual stimulus. The resulting data

Table 2

Corrected Group Mean Response Rates (Responses per Minute) During the Last Discrimination Training Session

\begin{tabular}{cccc}
\hline & \multicolumn{3}{c}{ Trial Type } \\
\cline { 2 - 4 } Group & VR & VT & Ext \\
\hline VR & 41.94 & 10.50 & 3.30 \\
VT & 29.49 & 0.90 & 0.66 \\
C & 32.40 & 3.78 & 1.32 \\
\hline
\end{tabular}

Note $-\mathrm{VR}=$ variable ratio, $\mathrm{VT}=$ variable time, Ext $=$ extinction, $\mathrm{C}=$ control.

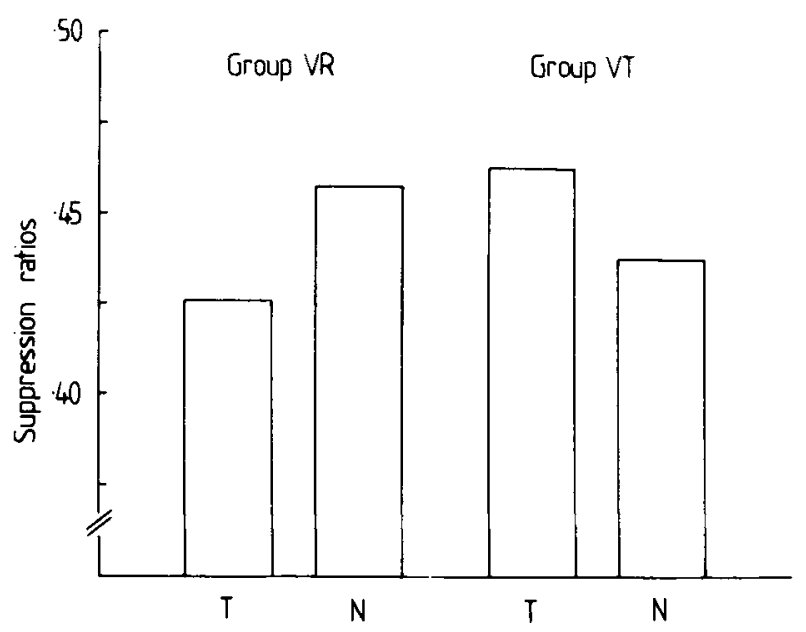

Figure 1. Group mean suppression ratios for Groups VR and VT, for trained (T) and novel (N) visual stimuli from the operant summation test.

are shown in Figure 1. They show that the visual stimulus used in discrimination training suppressed responding more than the novel visual stimulus in Group VR, but that in Group VT this pattern was reversed-the visual stimulus from training was actually less suppressive than its novel counterpart. (Subjects in Group $C$ received these test sessions in order to equate the experience of the groups prior to the final retardation test, but their data were not included in the analysis. In Group $C$, as in Group VT, the trained visual stimulus was less suppressive than the novel stimulus, although the difference between the two stimuli was smaller; the associated suppression ratios were .407 and .389 for trained and novel stimuli, respectively).

Planned orthogonal contrasts showed that the trained visual stimulus suppressed responding more than the novel visual stimulus in Group VR $[t(25)=2.12]$, but that there was no difference in the suppression produced by the trained and novel visual stimuli in Group VT $[t(25)=$ 1.73]. A three-way analysis of variance with group (VR or VT), training history of visual stimulus (trained or novel), and type of visual stimulus used in discrimination training (light-on or light-off) as factors was also performed on the ratio scores. There was no main effect of training history of the visual stimulus $(F<1)$, but there was a significant interaction between this factor and group $[F(1,25)=7.41]$. There was also a significant interaction between the training history of the visual stimulus and the type of visual stimulus used in discrimination training $[F(1,25)=5.90]$. No other effects or interactions were significant [largest $F(1,25)=1.59$ ].

Mean response rates during clicker trials were $48.22 \mathrm{rpm}$ for Group VR and $35.32 \mathrm{rpm}$ for Group VT. A two-way analysis of variance with group (VR or VT) and type of visual stimulus used in discrimination training (light-on or light-off) as factors revealed no significant effects or interactions [largest $F(1,25)=3.14$ ]. Hence, the differences in the ratio scores cannot be attributed to differences between the groups in responding during the clicker. 
The mean pretrial response rates for trials signaled by the clicker in compound with the visual stimulus used in training were $4.15 \mathrm{rpm}$ for Group VR and $1.80 \mathrm{rpm}$ for Group VT; the corresponding mean pretrial rates for trials signaled by the clicker in compound with the novel visual stimulus were $4.42 \mathrm{rpm}$ for Group VR and $1.93 \mathrm{rpm}$ for Group VT. A three-way analysis of variance with group (VR or VT), training history of visual stimulus (trained or novel), and type of visual stimulus used in training (light-on or light-off) revealed no main effect of the training history of the visual stimulus $(F<1)$. Although there was a significant interaction between the training history of the visual stimulus and the type of visual stimulus used in training $[F(1,25)=13.42]$, this did not interact with group $(F<1)$, and there were no other significant effects or interactions [largest $F(1,25)=3.34$ ]. Hence the pattern in the ratio scores cannot be attributed to differences in pretrial responding.

\section{Pavlovian Retardation Test}

The group mean corrected scores for the time spent in the magazine during stimulus presentations, pooled over the two test sessions, are presented in Figure 2. It shows that the trained visual stimulus came to elicit magazine behavior in Groups VR and C, whereas in Group VT it suppressed it. This suggests that the light was a Pavlovian inhibitor in Group VT but not in Group VR.

Nonparametric analysis of the corrected scores confirmed this description of the data. A set of orthogonal comparisons performed on the corrected scores showed that Group VR and Group $C$ did not differ $[U(8,14)=$ 43.5], but that these two groups combined did differ significantly from Group VT $(z=2.35, p<.02)$. This pattern cannot be attributed to differences in the pattern of pretrial responding. The mean pretrial scores, also pooled over the two test sessions, were $15.29 \mathrm{sec}$ for Group VR, $18.71 \mathrm{sec}$ for Group VT, and $12.84 \mathrm{sec}$ for Group C. A set of orthogonal comparisons performed on the pre-

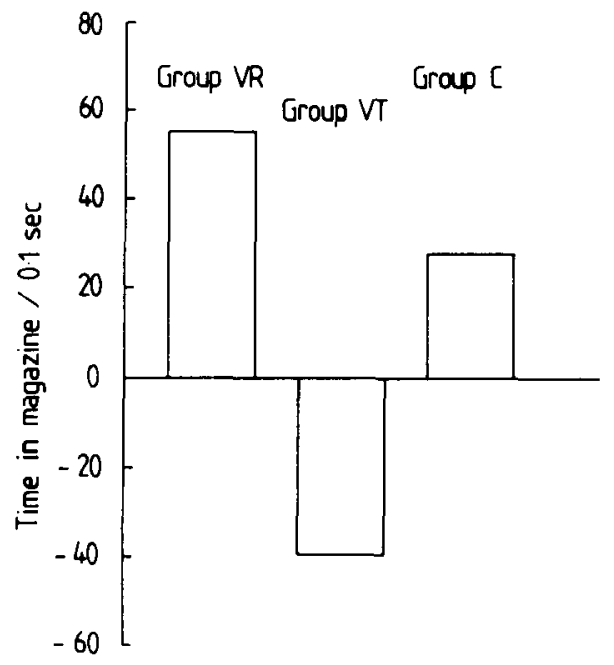

Figure 2. Group means of time spent in the magaxine during stimulus presentations, pooled over the Pavbovian retardation test sescions. Scores are corrected for pretrial responding. trial scores showed that Group VR did not differ from Group $C[U(8,14)=56]$, and that these two groups combined did not differ from Group VT $(z=0.124)$.

\section{DISCUSSION}

In Group VR the light suppressed operant responding, but there was no evidence that it was a Pavlovian inhibitor. Conversely, in Group VT the light was a Pavlovian inhibitor, and yet there was no evidence that it had any effect on operant responding. The first conclusion, therefore, is that Pavlovian inhibitors do not necessarily produce operant response suppression-in contrast to the findings of Gutman and Maier (1978). The second is that, since the light suppressed responding in Group VR but not in Group VT, this effect must be attributed to the fact that only in the former group was the light a signal for the absence of the contingency between responding and reinforcement. Neither of these findings can be accommodated by two-factor theory.

The failure to find an effect of Pavlovian inhibition stands in contradiction to Gutman and Maier's (1978) result, although it is consistent with a report by Lovibond (1983), who similarly found that an explicitly trained Pavlovian inhibitor had no effect on an operant response baseline. This apparent inconsistency may perhaps be resolved by suggesting that the suppression that Gutman and Maier's simple Pavlovian inhibitor produced could have been caused by nonassociative effects that were not adequately controlled for by their experimental procedure. Admittedly, the finding in the present experiment was a null result-but one might point out that, far from producing suppression, in numerical terms the light actually accelerated responding in Group VT. Moreover, given what is known about the effects of Pavlovian excitation on operant responding, the ineffectiveness of a Pavlovian inhibitor is perhaps not surprising. Two-factor theory also predicts that superimposing a Pavlovian excitor onto an operant response baseline should accelerate responding, and yet attempts to test this prediction have yielded inconsistent results. Although it is true that studies in which the operant response is no longer reinforced during the superimposition test usually find acceleration (e.g., Estes, 1943, 1948; Lovibond, 1981; Morse \& Skinner, 1958), if the instrumental reinforcement contingency is maintained during the test, suppression of responding is found (e.g., Azrin \& Hake, 1969; Karpicke, 1978; Karpicke, Christoph, Peterson, \& Hearst, 1977; Meltzer \& Brahlek, 1970; Stubbs, Hughes, \& Cohen, 1978) as often as acceleration (e.g., Edgar, Hall, \& Pearce, 1981; Herrnstein \& Morse, 1957; Lovibond, 1983; Meltzer \& Brahlek, 1970). If the effects of Pavlovian excitors are indeed so equivocal, the absence of an effect of inhibitors is less unexpected, and the adequacy of two-factor theory in accounting for discriminative control becomes increasingly questionable.

Far from suggesting that discriminative control can be accounted for in terms of Pavlovian conditioning, these data suggest that, on the contrary, the inhibitory control 
of operant responding is independent of Pavlovian inhibitory conditioning. In this respect they complement the view that, likewise, excitatory discriminative control may not depend on Pavlovian excitatory conditioning (e.g., Holman \& Mackintosh, 1981). On a wider level, the literature on negative occasion setting is also pertinent to this issue. An example of a negative occasion setter is the feature stimulus that operationally signals the absence of a contingency between a conditioned stimulus and a reinforcer in serial feature-negative discriminations; following its presentation, conditioned responding to the conditioned stimulus is reduced (e.g., Holland, 1985). Although such stimuli are operationally identical to Pavlovian conditioned inhibitors, there is evidence that they have properties unlike those traditionally associated with Pavlovian inhibition. Unlike Pavlovian inhibitors, they show evidence of extinction (Holland \& Gory, 1986), their properties are unaffected by Pavlovian excitatory conditioning (Holland, 1984), and they do not always transfer their inhibitory properties to other excitors (Holland \& Lamarre, 1984; for related work, see also Jenkins, 1985; Rescorla, $1985,1987)$. Because, like the light in Group VR, such stimuli may be viewed as signals for the absence of a contingency, albeit between a conditioned stimulus and a reinforcer rather than between a response and a reinforcer, it is tempting to suggest that their mechanism of action is the same. In the absence of further research, however, such a suggestion must remain speculative.

\section{REFERENCES}

Azrin, N. H., \& Hake, D. F. (1969). Positive conditioned suppression: Conditioned suppression using positive reinforcers as the unconditioned stimuli. Joumal of the Experimental Analysis of Behavior, $12,167-173$

Dickinson, A. (1985). Actions and habits: The development of behavioural autonomy. In L. Weiskrantz (Ed.), Animal intelligence (pp. 67-78). Oxford: Clarendon Press.

Edgar, D., Hall, G., \& Pearce, J. M. (1981). Enhancement of fcodrewarded instrumental responding by an appetitive conditioned stimulus. Quarterly Journal of Experimental Psychology, 33B, 3-19.

EsTEs, W. K. (1943). Discriminative conditioning: I. A discriminative property of conditioned anticipation. Journal of Experimental Psychology, 32, 150-155.

EsTES, W. K. (1948). Discriminative conditioning: II. Effects of a Pavlovian conditioned stimulus upon a subsequently established operant response. Joumal of Experimental Psychology, 38, 173-177.

Gutman, A., \& Maier, S. F. (1978). Operant and Pavlovian factors in cross-response transfer of inhibitory stimulus control. Learning \& Motivation, 9, 231-254.

Hearst, E., Besley, S., \& Farthing, G. W. (1970). Inhibition and the stimulus control of operant behavior. Journal of the Experimental Analysis of Behavior, 14, 373-409.

HeRRNSTEIN, R. J., MorSE, W. H. (1957). Some effects of responseindependent reinforcement on maintained operant behavior. Journal of Comparative \& Physiological Psychology, 50, 461-467.

Holland, P. C. (1984). Differential effects of reinforcement of an inhibitory feature after serial and simultaneous feature negative discrimination training. Joumal of Experimental Psychology: Animal Behavior Processes, 10, 461-475.
Holland, P. C. (1985). The nature of conditioned inhibition in serial and simultaneous feature negative discriminations. In R. R. Miller \& N. E. Spear (Eds.), Information processing in animals: Conditioned inhibition (pp. 267-297). Hillsdale, NJ: Erlbaum.

Holland, P. C., \& GoRY, J. (1986). Extinction of inhibition after serial and simultaneous feature negative discrimination training. Quarterly Joumal of Experimental Psychology, 38B, 245-265.

Holland, P. C., \& LamarRe, J. (1984). Transfer of inhibition after serial and simultaneous feature negative discrimination training. Learming \& Motivation, 15, 219-243.

Holman, J. G., Mackintosh, N. J. (1981). The control of appetitive instrumental responding does not depend on classical conditioning to the discriminative stimulus. Quarterly Journal of Experimental Psychology, 33B, 21-31.

Jenkins, H. M. (1985). Conditioned inhibition of keypecking in the pigeon. In R. R. Miller \& N. E. Spear (Eds.), Information processing in animals: Conditioned inhibition (pp. 327-354). Hillsdale, NJ: Erlbaum.

KARPICKE, J. (1978). Directed approach responses and positive conditioned suppression in the rat. Animal Leaming \& Behavior, 6, 216-224.

Karpicke, J., Christoph, G., Peterson, G., \& Hearst, E. (1977). Signal location and positive versus negative conditioned suppression in the rat. Journal of Experimental Psychology: Animal Behavior Processes, 3, 105-118.

LOVIBOND, P. F. (1981). Appetitive Pavlovian-instrumental interactions: Effects of inter-stimulus interval and baseline reinforcement conditions. Quarterly Journal of Experimental Psychology, 33B, 257-269.

LoviBond, P. F. (1983). Facilitation of instrumental behavior by a Pavlovian appetitive conditioned stimulus. Journal of Experimental Psychology: Animal Behavior Processes, 9, 225-247.

Meltzer, D., Brahlex, J. A. (1970). Conditioned suppression and conditioned enhancement with the same positive UCS: An effect of CS duration. Journal of the Experimental Analysis of Behavior, 13, 67-73.

Morse, W. H., \& SKINNER, B. F. (1958). Some factors involved in the stimulus control of operant behavior. Journal of the Experimental Analysis of Behavior, 1, 103-107.

Pfautz, P. L., Donegan, N. H., \& Wagner, A. R. (1978). Sensory preconditioning versus protection from habituation. Journal of Experimental Psychology: Animal Behavior Processes, 4, 286-295.

Rescorla, R. A. (1969). Pavlovian conditioned inhibition. Psychological Bulletin, 72, 77-94.

RescorLA, R. A. (1985). Conditioned inhibition and facilitation. In R. R. Miller \& N. E. Spear (Eds.), Information processing in animals: Conditioned inhibition (pp. 299-326). Hillsdale, NJ: Erlbaum.

Rescorla, R. A. (1987). Facilitation and inhibition. Journal of Experimental Psychology: Animal Behavior Processes, 13, 250-259.

Rescorla, R. A., Solomon, R. L. (1967). Two-process learning theory: Relationships between Pavlovian conditioning and instrumental learning. Psychological Review, 74, 151-182.

SPENCE, K. W. (1936). The nature of discrimination learning in animals. Psychological Review, 43, 427-449.

Stubrs, D. A., Hughes, J. E., Cohen, S. L. (1978). Positive conditioned suppression: An explanation in terms of multiple and concurrent schedules. Journal of the Experimental Analysis of Behavior, 30, 329-343.

Trapold, M. A., \& Overuarr, J. B. (1972). The second learning process in instrumental learning. In A. H. Black \& W. F. Prokasy (Eds.), Classical conditioning II: Current research and theory (pp. 427-452). New York: Appleton-Century-Crofts.

(Manuscript received November 3, 1987; revision accepted for publication March 1, 1988.) 